\title{
Critical steps in the early evolution of the isocortex. Insights from developmental biology
}

F. Aboitiz,

J. Montiel and

J. López
Departamento de Psiquiatría, Facultad de Medicina y Centro de Investigaciones Médicas, Pontificia Universidad Católica de Chile, and Millenium Nucleus for Integrative Neuroscience, Santiago, Chile

\section{Correspondence \\ F. Aboitiz \\ Departamento de Psiquiatría \\ Facultad de Medicina \\ Universidad Católica de Chile \\ Marcoleta No. $387,2^{\circ}$ piso \\ Casilla 114-D \\ Santiago 1 \\ Chile \\ Presented at the IV International UNESCO Course on "What the \\ Developing Cerebral Cortex Tells About the Adult Cortex (and Vice Versa)", Rio de Janeiro, RJ, Brazil, December 3-7, 2001. \\ Research partially supported by Fondecyt (No. 1970294), and by the Millenium Nucleus for Integrative Neuroscience. J. Montiel was the recipient of a Conicyt doctoral fellowship.}

Received July 12, 2002 Accepted October 7, 2002 $\ldots \ldots \ldots \ldots \ldots \ldots \ldots$
Abstract

This article proposes a comprehensive view of the origin of the mammalian brain. We discuss i) from which region in the brain of a reptilian-like ancestor did the isocortex originate, and ii) the origin of the multilayered structure of the isocortex from a simple-layered structure like that observed in the cortex of present-day reptiles. Regarding question i there have been two alternative hypotheses, one suggesting that most or all the isocortex originated from the dorsal pallium, and the other suggesting that part of the isocortex originated from a ventral pallial component. The latter implies that a massive tangential migration of cells from the ventral pallium to the dorsal pallium takes place in isocortical development, something that has not been shown. Question ii refers to the origin of the six-layered isocortex from a primitive three-layered cortex. It is argued that the superficial isocortical layers can be considered to be an evolutionary acquisition of the mammalian brain, since no equivalent structures can be found in the reptilian brain. Furthermore, a characteristic of the isocortex is that it develops according to an inside-out neurogenetic gradient, in which late-produced cells migrate past layers of early-produced cells. It is proposed that the inside-out neurogenetic gradient was partly achieved by the activation of a signaling pathway associated with the Cdk5 kinase and its activator $\mathrm{p} 35$, while an extracellular protein called reelin (secreted in the marginal zone during development) may have prevented migrating cells from penetrating into the developing marginal zone (future layer I).

\section{Introduction}

This paper is concerned with the early stages of mammalian brain evolution. Our strategy to approach this issue will be to borrow evidence from developmental biology and from neuroanatomy in order to validate or reject proposals of homology and evolutionary mechanisms. In order to do so, we will first very briefly discuss the phylogenetic relationships of terrestrial vertebrates to show the position of mammals in relation to birds and
Key words

- Cdk5

- Dorsal ventricular ridge

- Reelin

- Tangential migration

- Ventral pallium reptiles; then, we will discuss brain organization in birds, reptiles and mammals. After this introduction, the paper will consist of two parts. In the first part, we address the issue of structures homologous to the mammalian isocortex. This section includes evidence derived from brain connectivity and from gene expression during early development. The second part addresses the origin of isocortical lamination, and will be largely based on the analysis of cortical migration mutants. We will also describe a scenario that intends to make func- 
tional sense of the generation of the inverted, inside-out neurogenetic gradient of the mammalian isocortex.

\section{Phylogenetic relationships and differences in brain structure}

Figure 1 indicates a family tree, or cladogram of different vertebrates, together with a diagram of their cerebral hemispheres. As can be seen, amphibians are the sister group of mammals, birds and reptiles all together; mammals are the sister group of birds and reptiles. Note that birds are not the sister group of reptiles. Instead, birds belong to reptiles, and the structure of their brain reflects this phylogenetic position. This paper will be concerned with the transition between the common ancestor of mammals and reptiles, and modern mammals.

The first reptiles to appear in the fossil record, called anapsids (also termed stem reptiles), probably gave rise to all present-day reptiles, including birds (1). Another group of early reptiles, called synapsids, were the ancestors of the first mammals. The relationship of the synapsids with the stem reptiles is rather obscure. Interestingly, the cranial cavity of both anapsids and synapsids was small and narrow, suggesting that they had a small brain (2). In both lines, primitive reptiles and mammal-like reptiles, the expansion of the brain was a relatively late event. In the mammalian line, the brain became enlarged only when the first true mammals appeared, and was more or less simultaneous with the origin of the middle ear $(3,4)$. Therefore, the expansion of the mammalian brain took place as a very fast evolutionary step, and relatively late in the history of these animals. This is important because it indicates that the enlargement of the brain may have occurred independently in the line leading to reptiles and in the line leading to mammals. Thus, one important question that we will address in this paper, from the perspective of comparative neuroanatomy, is to what extent it is correct to consider mammals as derived from reptiles as we know them today.

Before we deal with the main questions, it will be useful to recall some general aspects of comparative brain anatomy. Figure 1 schematically illustrates the structure of the brain in the main classes of terrestrial vertebrates. The brains of amphibians have the simplest organization of all vertebrates and possibly resemble the ancestral amniote brain. Amphibian brains have no large cell masses, and most cells are located in the periventricular
Figure 1. Phylogenetic relations of terrestrial vertebrates. In each taxon, one cerebral hemisphere of a representative species is depicted. Medial is to the left of the observer. DCx, dorsal cortex; DP, dorsal pallium; DVR, dorsal ventricular ridge; HP, hippocampus; LCX, lateral cortex; LP, lateral pallium; $\mathrm{MCx}$, medial cortex; MP, medial pallium; STR, corpus striatum; $\mathrm{OCx}$, olfactory cortex; ICx, isocortex.

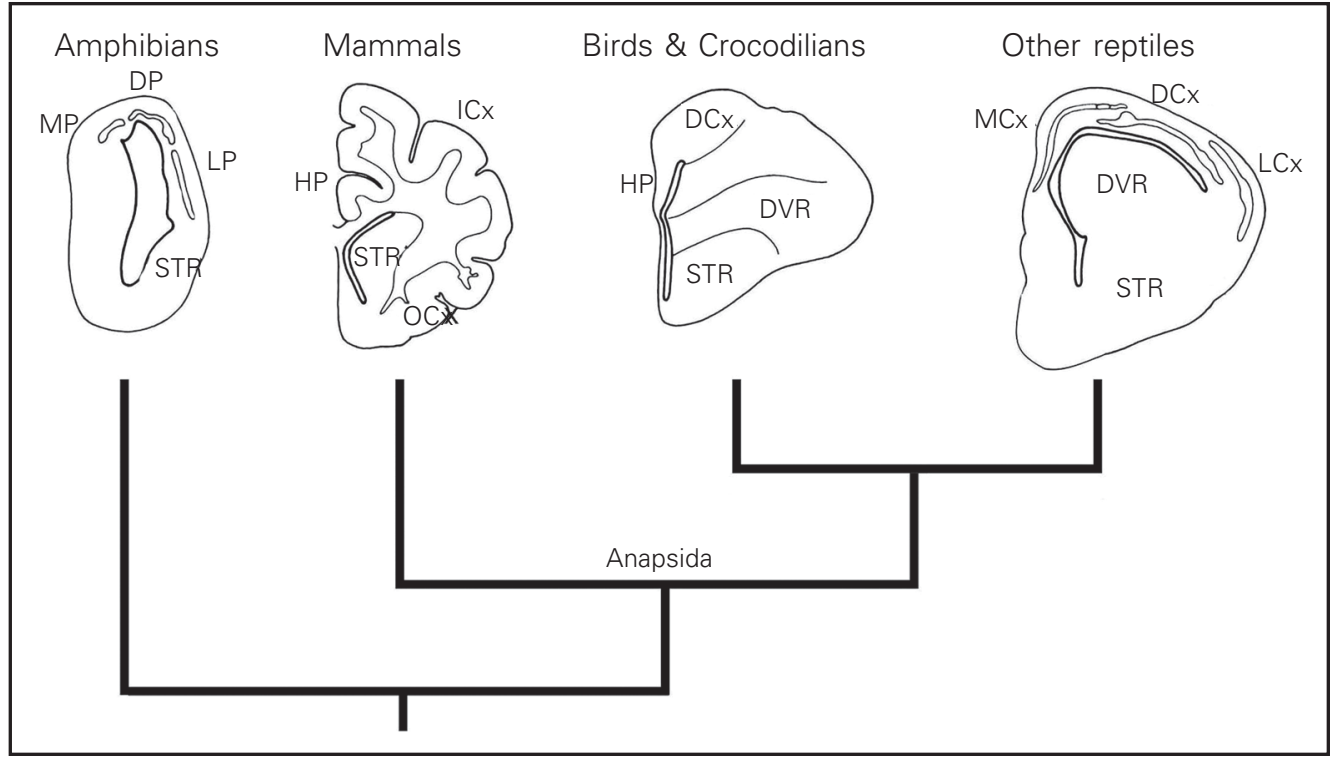


zone, there being little cell migration away from the latter. Thalamic sensory projections terminate mostly in the medial pallium and in the corpus striatum (5). The brains of reptiles have a small, three-layered cerebral cortex consisting of a medial part and a dorsomedial part, both of which make up the homologue of the hippocampal formation of mammals; a lateral part which corresponds to the olfactory cortex of mammals, and a very small dorsal cortex, which is possibly homologue to the isocortex. In reptiles, thalamic sensory afferents terminate in the dorsal cortex and in a periventricular structure that bulges into the lateral ventriculum and is located immediately above the corpus striatum, called the dorsal ventricular ridge (DVR). The DVR (especially its anterior part, which receives most sensory projections) is the main sensory processing center of the reptilian brain (6). Finally, mammals are characterized by the presence of a six-layered neocortex or isocortex, located between the hippocampal (medial) cortex and the olfactory (lateral) cortex. The isocortex receives most thalamic sensory projections, and expands significantly in rela- tion to other brain components.

We will address two main questions in this paper. The first is, which is the structure ancestral to the isocortex in the reptilian brain?, and the second is, how did the isocortex acquire its six-layered organization? The first question is related to the topographic position of the isocortex (and its ancestral structure) in the brain of early reptiles or mammal-like reptiles, while the second question is related to the evolution of the internal structure of the isocortex. In this context, it is important to remember that the isocortex has a conserved laminar organization among mammals, even though it may expand significantly in some groups like primates. Our second question therefore is related to the steps involved in the acquisition of the six-layered organization that is common to the isocortex of most mammals.

\section{Question 1. Homologues to the isocortex}

There have been two main hypotheses about the homologies to the isocortex in the non-mammalian brain (Figure 2). The first

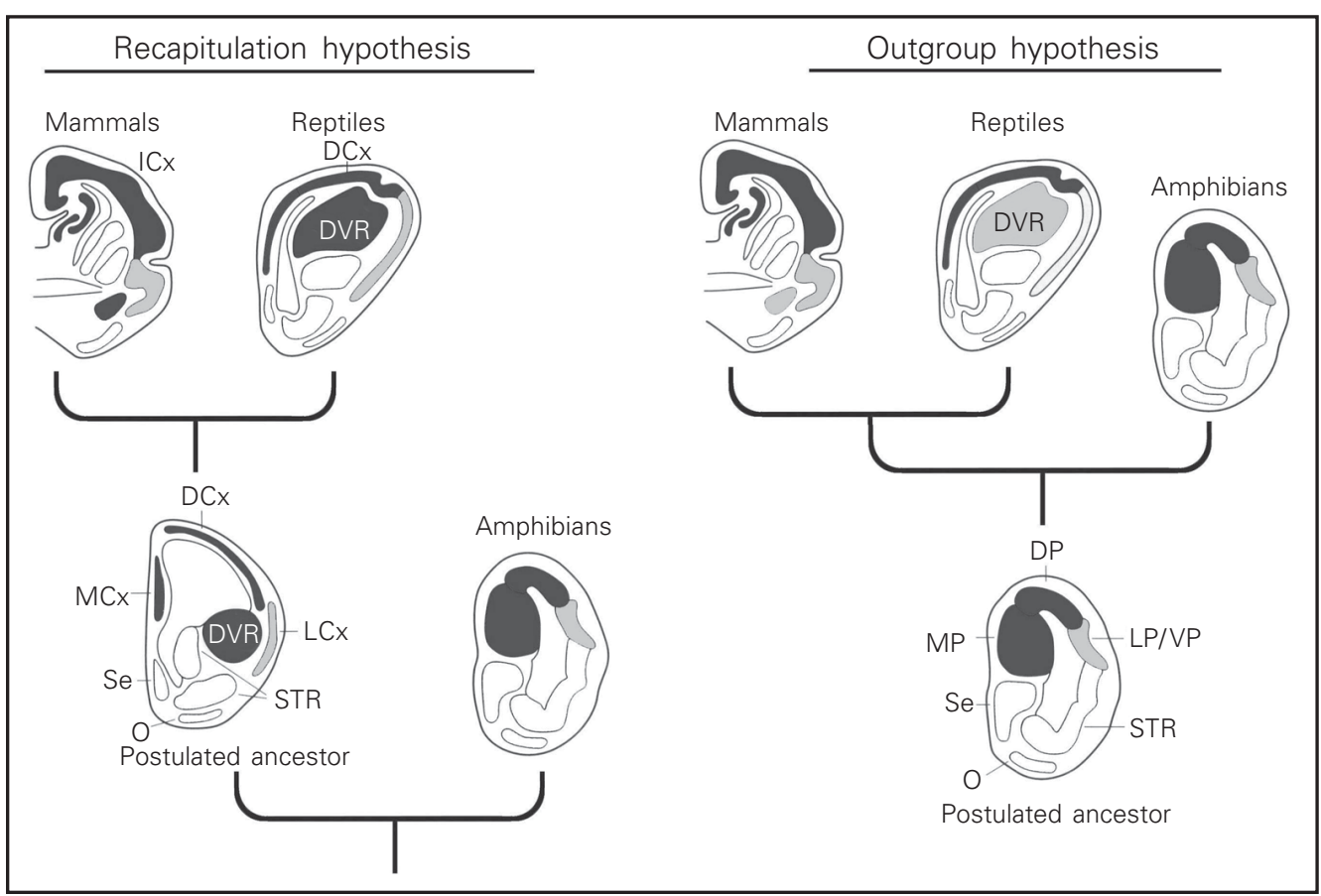

Figure 2. Diagrams summarizing the essential elements of the recapitulation hypothesis and the outgroup hypothesis. O, olfactory tubercle; Se, septum; VP, ventral pallium. For other abbreviations, see legend to Figure 1 
hypothesis, which was termed the "recapitulation hypothesis" by Northcutt and Kaas (7), asserts that the common ancestor of mammals and reptiles had a reptilian-like brain, with a DVR (or its precursor), and a dorsal cortex. This hypothesis implies that the DVR, together with the dorsal cortex, gave rise to the mammalian isocortex. The second hypothesis, the "outgroup hypothesis" (7), asserts that the common ancestor of mammals and reptiles had a very simple brain, similar to that of amphibians, with no large cell masses. According to this view, only the dorsal cortex of this common ancestor became the isocortex. Thus, the main difference between the two hypotheses is whether the DVR of reptiles did or did not give rise to the isocortex.

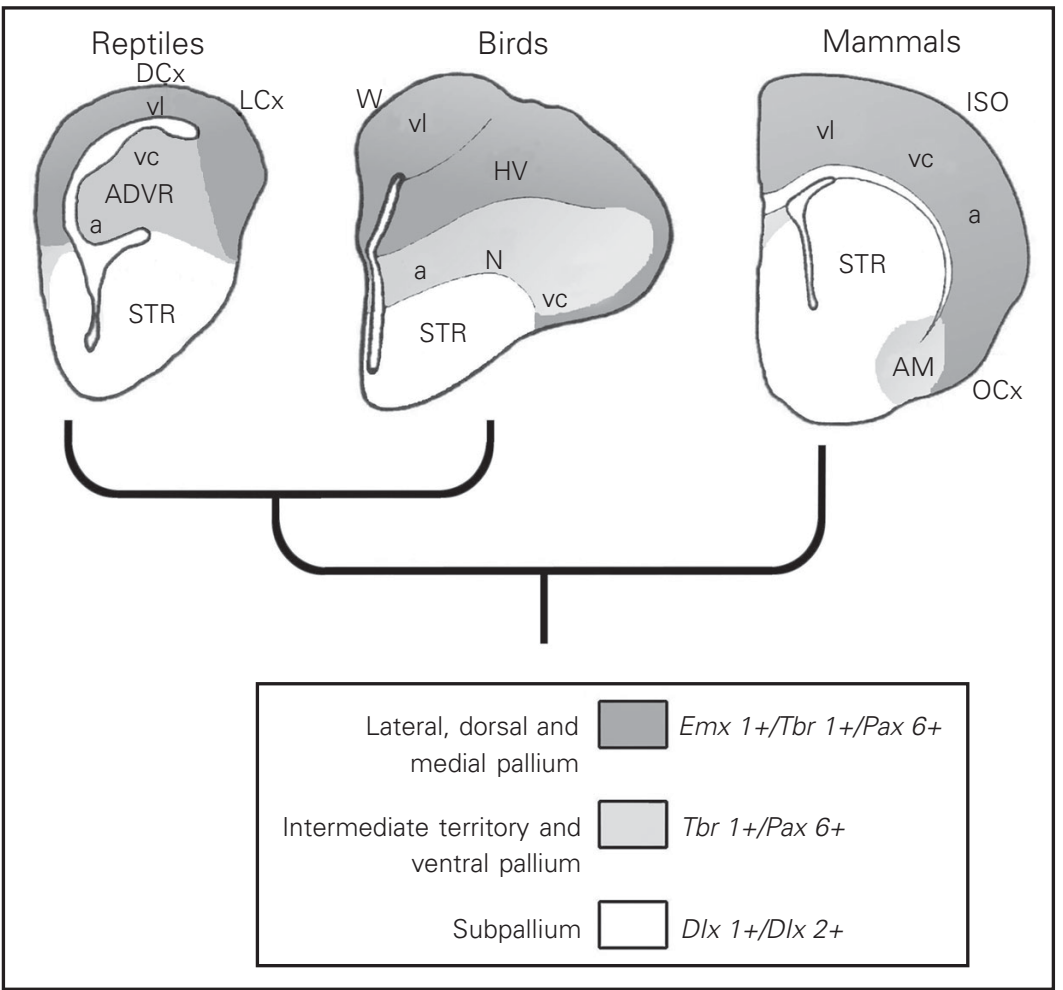

Figure 3. This figure indicates two different schemes. One is the site of termination of visual thalamofugal or lemniscal (vl) pathways, of visual tectofugal or collothalamic (vc) pathways, and of auditory (a) pathways, in the cerebral hemispheres of different vertebrates. Additionally, the Figure indicates the dorsoventral subdivision of the amniote hemisphere into three main components: i) lateral, dorsal and medial pallium, ii) ventral pallium, and iii) subpallium. Each component is defined by the expression of a specific group of regulatory genes. ADVR, anterior dorsal ventricular ridge; AM, amygdala; HV, hyperstriatum ventrale; ISO, isocortex; N, neostriatum, a component of the avian DVR; W, Wulst, equivalent to the reptilian dorsal cortex. For other abbreviations, see legend to Figure 1.

\section{Recapitulation hypothesis}

This hypothesis relies mostly on evidence of the termination of thalamic sensory pathways in the telencephalon. A good example to illustrate this evidence is the visual system. In most vertebrates, there are two visual pathways. One is the thalamofugal pathway, which in mammals originates from retinal projections to the lateral geniculate nucleus; the pathway then follows to the primary or striate visual cortex ("vl" in Figure 3). The second pathway, the tectofugal pathway ("vc" in Figure 3), goes from the retina to the superior colliculus or optic tectum, then projects to the pulvinar nucleus in the thalamus, and finally projects to the extrastriate visual cortex. The same two pathways can be observed in reptiles and birds, and in most vertebrates. In birds and reptiles, the thalamofugal pathway ("vl" in Figure 3) is relayed in the nucleus equivalent to the lateral geniculate, and terminates in the dorsal cortex (the Wulst of birds). On the other hand, the tectofugal pathway ("vc" in Figure 3) projects to the optic tectum, then projects to a thalamic nucleus called nucleus rotundus, and from there it is directed to the DVR $(8,9)$.

Based on this evidence, it has been proposed that the thalamofugal pathway, which ends in the dorsal cortex or Wulst of birds, and in the striate cortex of mammals, indicates homology between the striate cortex and the dorsal cortex or Wulst. On the other hand, the tectofugal pathway, terminating in the DVR and in the extrastriate visual cortex, indicates homology between these two structures. It was also claimed that the nucleus rotundus was homologous to the mammalian pulvinar nucleus because they are located at corresponding processing stages in the tectofugal pathway. Furthermore, considering similarities in intrinsic connectivity, it was postulated that the different laminae of the extrastriate visual cortex might correspond to different components of the 
avian DVR. Thus, there was an equivalent circuitry in the mammalian cortex and in the avian and reptilian DVR, which probably was ancestral to both groups. In addition to this evidence, the finding that the auditory pathway ("a" in Figure 3) ends in the avian DVR suggested that the mammalian auditory cortex derived from a DVR-like structure $(8,9)$.

More recently, Butler (10) proposed a subdivision of the thalamic nuclei that somehow agrees with the above view. She classified thalamic sensory nuclei as either "collothalamic" or "lemnothalamic" (Table 1). Collothalamic nuclei receive sensory projections from the mesencephalon. The pulvinar nucleus of mammals and the nucleus rotundus of birds, both of which receive the visual tectofugal pathway, and the medial geniculate and its reptilian equivalent, receiving the auditory pathway, are proposed to be examples of collothalamic nuclei. On the other hand, lemnothalamic nuclei receive projections from lemniscal pathways which bypass the mesencephalon. Examples are the lateral geniculate nucleus, receiving the visual thalamofugal pathway, and the somatosensory nuclei receiving the spinothalamic pathway.

Collothalamic nuclei project to the DVR of reptiles and birds, and to what we call the ventrolateral isocortex of mammals. This includes the visual extrastriate isocortex and the auditory isocortex. At the same time, lemnothalamic nuclei project to the dorsal cortex of reptiles and birds, and to what we call the dorsomedial isocortex of mammals, which includes the visual striate isocortex and the somatosensory isocortex of mammals. Overall, considering this connectional evidence, the ventrolateral isocortex, including the extrastriate and auditory isocortex, would be homologous to the anterior DVR, since both receive collothalamic projections. The dorsomedial isocortex, including the striate isocortex and somatosensory isocortex, would be homologous to the dorsal cortex of reptiles and birds, since these structures receive lemnothalamic projections. This recapitulationist hypothesis implies that the sensory projection sites and processing circuits have been conserved in reptiles and in mammals, while there have been gross changes in brain topography and cytoarchitecture.

\section{Outgroup hypothesis. I. Connectional evidence}

Homology of the striate cortex and the somatosensory cortex with the avian or reptilian dorsal cortex is well accepted. The Wulst (dorsal cortex) of the pigeon contains regions that can be compared with the mammalian primary (or striate) visual cortex, with the primary somatosensory cortex and

Table 1. Subdivisions of thalamic nuclei (10).

\begin{tabular}{lll}
\hline Thalamic subdivision & Input and nucleus & Projection \\
\hline Collothalamus & Visual tectofugal & ADVR (reptiles, birds) \\
(collicular pathways) & (pulvinar, rotundus) & VL (extrastriate) isocortex (mammals) \\
& Auditory & ADVR (reptiles, birds) \\
& (medial geniculate) & VL (auditory) isocortex (mammals) \\
& & \\
Lemnothalamus & Visual thalamofugal & Dorsal cortex (reptiles, birds) \\
(lemniscal pathways) & (lateral geniculate) & DM (striate) isocortex (mammals) \\
& Somatosensory & Dorsal cortex (reptiles, birds) \\
& (ventral posterior) & DM (somatosensory) isocortex (mammals)
\end{tabular}

ADVR, anterior dorsal ventricular ridge; $\mathrm{VL}, \mathrm{DM}$, ventrolateral and dorsomedial isocortex, respectively. 
with a region that might be considered a mixture of somatosensory and motor cortex (11). However, the concept of homology between the DVR and the isocortex has proved to be more controversial. Some early questions regarding this hypothesis $(12,13)$ took issue with the topography of the reptilian brain. The position of the DVR in the hemisphere is such that in most reptiles it is separated from the dorsal cortex by the lateral cortex (Figure 2). Therefore, the DVR would somehow have to move to a more dorsal position in order to make up part of the isocortex.

Additional conflicting evidence comes from neuronal connectivity. In amphibians, collothalamic projections end mostly in the corpus striatum (14), which is clearly not a homologue of the DVR or the isocortex of mammals. This indicates that collothalamic pathways may change their preferred termination sites, and therefore they may not be considered a good criterion to establish homology. Furthermore, Bruce and Neary (15) and later Puelles and collaborators $(16,17)$ have indicated that the overall pattern of connections of the DVR resembles more the mammalian laterobasal amygdala than the isocortex. Collothalamic projections also terminate in the laterobasal amygdala of mammals, but instead of arising from specific nuclei like the pulvinar nucleus, they originate from the intralaminar thalamic nuclei (Figure 4). Thus, the conclusion from these studies has been that the intralaminar thalamic nuclei, and not the mammalian pulvinar, are comparable to the avian nucleus rotundus.

Finally, another source of evidence has to do with the connections of the extrastriate cortex. This is very old evidence but it has never been considered enough in this context. In mammals, the striate visual cortex connects strongly to the extrastriate visual cortex, while in reptiles there is little connectivity between the dorsal cortex and the DVR (6). Furthermore, in mammals, the extrastri- ate and the auditory cortices project, via a sequence of cortico-cortical connections, to the entorhinal cortex and to the hippocampus. On the other hand, in reptiles the anterior DVR (receiving thalamic sensory afferents) projects to the posterior DVR (also termed the "reptilian amygdala") (6), but is poorly connected to the hippocampus (18-20).

\section{Outgroup hypothesis. II. Developmental evidence}

Some relatively recent reports (21-23) concerning the topography of gene expression patterns in the early development of the telencephalon revealed a highly conserved, mosaic-like, organization of the different telencephalic components (pallium and subpallium). A novel component, located in the equatorial region of the hemispheres, was described in these studies which was termed intermediate territory (21) or, perhaps more appropriately, ventral pallium $(22,23)$. The ventral pallium gives rise to the DVR (especially the anterior part) of reptiles and to the lateral basal amygdala of mammals, which implies that these adult structures derive from the same embryonic region. This is a strong argument in favor of homology between the DVR and parts of the mammalian amygdala. Interestingly, based on purely descriptive considerations, Holmgren (24) had already considered that the amygdalar complex was comparable to the DVR of reptiles, and he termed these two things the hypopallium.

Figure 3 shows the molecular evidence dividing the cerebral hemispheres into three main sectors. During embryogenesis, the lateral, dorsal, and medial pallium are all positive for the same subset of homeotic-like genes, such as Emx 1, Tbr 1, and Pax 6. The subpallium, which corresponds to the corpus striatum and other ventral structures, is positive for the genes Dlx 1 and Dlx 2. The ventral pallium is located in the equatorial region of the hemisphere, between the pal- 
lium and subpallium. This territory has pallial markers like Pax 6 and Tbr 1, but does not express the Emx 1 marker. Apparently, the ventral pallium has a different fate in the development of the mouse and the chick (21). In the mouse, the ventral pallium disappears relatively soon in development, and gives rise only to early-produced neural populations. However, in the chick the ventral pallium may expand and give rise to a large part of late-produced elements of the DVR.

Summarizing these results, the avian and reptilian DVR is comparable to parts of the amygdala of mammals (these two make up the ventral pallium in reptiles and mammals, respectively), and the dorsal cortex of reptiles and birds is embryologically comparable to the mammalian isocortex (both derive from the dorsal pallium). A consequence of this conclusion is that in mammals, collothalamic projections reach the dorsal pallium, while in reptiles, the same projections terminate in the ventral pallium. Therefore, this hypothesis implies that there has been an important change in brain connectivity in the origin of mammals.

\section{Tangential migration}

The developmental evidence about gene expression patterns strongly confirms the concept based on brain topography, indicating that the lateral cortex is interposed between the dorsal cortex and the DVR. As mentioned above, in order to be correct the recapitulation hypothesis requires that during development the DVR be displaced dorsalwards, merging with the developing dorsal cortex. May cells from the ventral pallium migrate into the dorsal pallium and contribute to the isocortex? There is now substantial evidence that $D l x$-positive GABAergic cells from the embryonic ganglionic eminences may migrate into the isocortex $(25,26)$, and that many pioneer neurons of the preplate possibly migrate tangentially from the ganglionic eminences (27). However, the ganglionic eminences do not correspond to the ventral pallium, but rather belong to the embryonic subpallium. Sec-

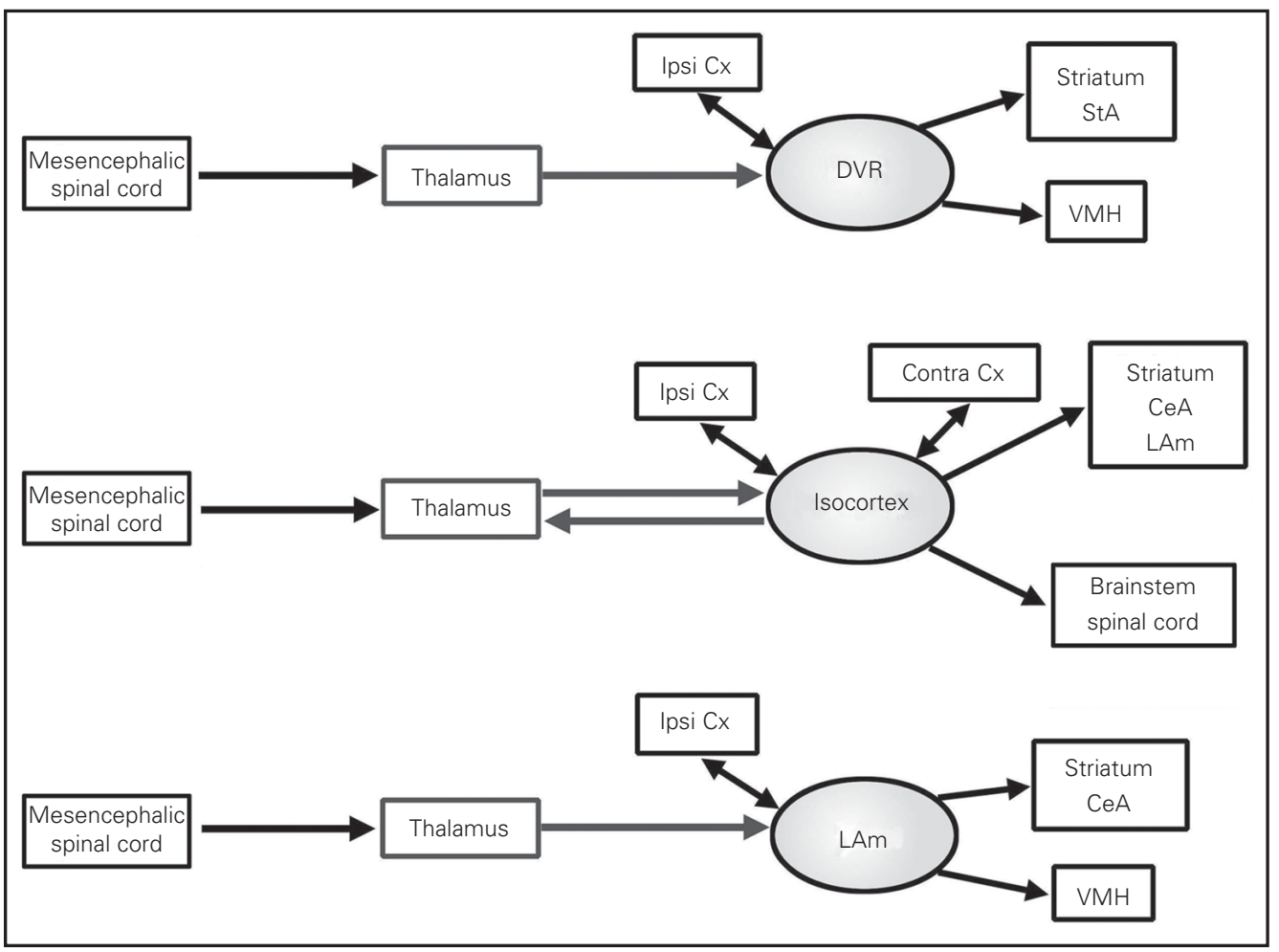

Figure 4. Schematic patterns of connectivity of the reptilian dorsal ventricular ridge (DVR), the isocortex and the lateral amygdala (LAm). CeA, central amygdala; StA, striatal amygdala; $\mathrm{VMH}$, ventromedial hypothalamus; Cx, cortex. 
ond, the kind of cells that migrate, mostly GABAergic interneurons, do not correspond to those specified by the equivalent circuit hypothesis (this implies only excitatory elements) (8). No evidence has been found as yet for a massive migration of excitatory cells from the ventral pallium into the dorsal pallium, as the recapitulation hypothesis would imply.

The $D l x$-positive cells that migrate from the ganglionic eminence into the isocortex keep expressing their subpallial marker. Likewise, if there was such a migration from the ventral pallium into the isocortex, this might make the auditory and extrastriate visual cortical areas largely negative to $E m x$, a fact that has not been observed. Recent evidence indicates that Emx 1 is expressed in most pyramidal cells of the isocortex (28), which is not expected if some cells arise from the Emx 1negative ventral pallium. Furthermore, in dissociated cortical cultures, this gene is expressed by most glutamate-containing neurons (28), suggesting that the (excitatory) cells that make up the equivalent circuits in mammals and birds $(8,9)$ may express different markers (they are Emx 1-positive in the isocortex and Emx 1negative in the DVR). It could still happen that some precursor cells migrate from the ventral pallium very early into the dorsal cortex, and then they acquire Emx 1 expression. To be fair, it would be very hard to prove that this process does not occur. Undoubtedly, the incorporation of GABAergic cells from the subpallium was probably an important element in isocortical origins. Nevertheless, this sort of migration of inhibitory interneurons has been observed in birds (29), suggesting that this mechanism predates the origin of mammals.

\section{A new type of brain}

The evidence reviewed suggests that in mammals a noticeable expansion of the dorsal pallium has taken place, perhaps to some extent at the expense of the development of the ventral pallium. On the other hand, in reptiles the ventral pallium is the structure that expands most, while the dorsal pallium remains relatively small. Therefore, we may perhaps speak of a "dorsalized" pallium in mammals, as opposed to a "ventralized" pallium in reptiles and birds. In this context, an interesting example is the small eye mutation in mice, which renders the Pax 6 gene inactive. In this mutant, there is a dorsal displacement of the domains of expression of many genes characteristic of subpallial sectors, i.e., there is a dorsal displacement of the pallial-subpallial boundary $(30,31)$. For example, $D l x 1$ genes are normally expressed mainly in the medial and lateral ganglionic eminence. However, in this mutant, they invade pallial territory, which acquires a subpallial identity. It is possible that in the evolution of the pallium, similar shifts in the territories of gene expression have occurred, partly giving rise to the different patterns of brain organization that we see in reptiles and mammals. Perhaps subtle shifts in the relative emphasis on dorsalizing versus ventralizing factors in telencephalic development may produce important changes in brain organization. Nevertheless, both in the reptilian and in the mammalian lineages there has been a parallel tendency to an increase in brain size (in reptiles mostly in the ventral pallium, and in mammals in the dorsal pallium), which may not be explained by a mere shift in compartmental boundaries. It is possible that the overexpression of some factor(s) promoting dorsal pallial growth in mammals, or ventral pallial growth in reptiles might perhaps account for the differential growth of each of these pallial components, respectively. In this context, it has been recently found that the protein Sonic hedgehog is required for dorsal expansion of the developing midbrain and forebrain (32). Perhaps signals of this sort may regulate the expansion of different telencephalic components, and their differential regulation might produce diverging phenotypes that might lead to different patterns of brain organization such as those observed in reptiles and mammals. 
Another important point raised by the evidence reviewed is that this implies different patterns of brain connectivity between reptiles and mammals. In reptiles, the collothalamic projections and the lemnothalamic projections are largely separate. Collothalamic input enters the DVR, which is mainly connected to the hypothalamus, while the lemnothalamic input enters the dorsal cortex, which projects to the hippocampus, and establishes association networks with the olfactory cortex (6). On the other hand, in mammals there is a confluence of collothalamic and lemnothalamic inputs, especially in the visual system (there is a strong projection from the striate cortex to the extrastriate cortex), and both the lemno- and collothalamic pathways project to the hippocampus and the amygdala. Summarizing, in reptiles there seems to be a separation of the collothalamic and the lemnothalamic processing systems, the former related to the amygdala and the latter to the hippocampus. In mammals, both collothalamic and lemnothalamic projections end in the dorsal pallium, and from there both pathways project to the hippocampus and to the amygdala.

\section{The role of olfaction in the origin of the mammalian brain}

What adaptive mechanisms and functional constraints may have driven the change in brain organization in the evolution of mammals? One possibility is that olfaction, which was a highly developed sense in the early mammals (2), was a key factor in determining a different evolutionary direction in mammalian brain evolution. In both mammals and reptiles, the olfactory system is highly connected to the hippocampus (medial cortex); furthermore, the dorsal cortex of reptiles receives olfactory projections and sends projections to the medial cortex (hippocampus) (6). Thus, the dorsal cortex appears as an important element in olfactoryhippocampal interactions. In addition, the dorsal cortex and the hippocampus of reptiles participate in spatial learning, and can make use of non-spatial cues (like odors and other stimuli) in orientation (33). Considering the relative importance of olfaction in the behavior of early mammals, we have proposed (12) that the olfactory-hippocampal-dorsal cortex circuit may have been put to use by the first mammals to make relatively elaborate, largely olfactory-based, representations of behavioral space, in which specific odors labeled particular places and routes. In mammals, there is strong evidence that odor information participates in spatial and episodic learning (34), and these may have been important functions of olfaction in the first mammals. Nevertheless, the contribution of the visual system became undoubtedly helpful in the elaboration of more precise maps of space, especially when mammals invaded diurnal niches after the decline of dinosaurs. The dorsal cortex, receiving visual information from the thalamofugal visual pathway, may have become an important sensory processing system in the early mammalian brain (12). This may have triggered expansion of this structure, and the eventual incorporation of sensory information from collothalamic systems.

It is of interest to note that in reptiles, visual spatial information is processed mainly via the collothalamic, tectofugal visual pathway, while in mammals the lemnothalamic, thalamofugal pathway conveys most of the spatial information (35). Likewise, auditory information - also related to collothalamic pathways - has an important spatial component, and there is a strong collothalamic somatosensory pathway in reptiles (36). Thus, in reptiles spatial information from most sensory sources is conveyed by mesencephalic components and their associated collothalamic pathways. Furthermore, as mentioned, the hippocampus of reptiles receives fewer projections from the collothalamic pathways compared to mammals. We propose that one main innovation in the origin 
of the mammalian brain is the confluence of the collothalamic and the lemnothalamic pathways in order to process spatial information which, among other things, participated in spatial learning and episodic memory. In this process, the hippocampus may have become a fundamental component in which both types of sensory pathways eventually converged. Strictly speaking, this particular proposal is consistent with both the recapitulation and the outgroup hypotheses, since the merging of the two pathways is independent of the embryonic origin of the ventrolateral isocortex. However, it may be more parsimonious to consider that for this confluence to occur, only the axonal projections changed their route instead of producing a massive cellular migration that dragged the collothalamic axons to a more dorsal position. Perhaps a middle-way possibility would be that only some cells, recipient of thalamic afferents in the ventral pallium, migrated dorsally to the dorsal pallium, carrying with them the thalamic afferents. This would not need a massive tangential migration, and many components of the equivalent circuits (9) would be derived from the dorsal pallium in mammals. However, as mentioned, there is no evidence for this migratory process.

\section{Question 2. Laminar development of the isocortex}

The second part of this paper relates to the internal structure of the isocortex. There are a number of differences between the reptilian cortex and the mammalian isocortex. First, the reptilian cortex is much smaller, has three layers, and has a tangential organization of inputs in layer I, above the cortex. The mammalian isocortex is a highly expansive structure, has 6 layers, and afferents come radially from the white matter underneath (6). Second, the reptilian cortex develops according to an outside-in neurogenetic gradient, in which early-produced neurons are located above late-produced neurons (37); in the isocortex there is an inside-out neurogenetic gradient in which early-produced cells are located in inferior layers, while subsequently produced cells are located in more superficial layers (38). In the following sections, we will review some morphological and genetic aspects of cortical development in order to propose some plausible evolutionary scenarios for the origin of the developmental and structural characteristics of the isocortex.

\section{Comparative cortical development}

As a brief reminder, mammalian isocortical development is characterized by the presence of an early preplate, which is a population of cells that is subsequently divided into a deep subplate and a superficial marginal zone by the arrival of cells belonging to the cortical plate. Most of these preplate cells die in late cortical development. A characteristic cell type of the marginal zone is the Cajal-Retzius cell which secretes reelin, an extracellular protein involved in the control of neuronal migration $(39,40)$.

One unsolved question is whether there is a preplate in the reptile. An early population of somatostatin-positive and NPY-positive cells has been detected in the developing cortex of reptiles $(40,41)$. However, these cells do not express reelin or calbindin, which are markers of the mammalian preplate. If there is something like a preplate in the reptiles, it has important differences with what we see in the mammal. Furthermore, it is not known whether the preplate is split by the arrival of the cortical plate, leaving a subplate-like structure underneath.

Marín-Padilla (42) originally proposed that the mammalian subplate represented the ancestral reptilian cortex. However, the subplate evidences a progressive elaboration in those mammals with more complex isocortices, which suggests that more than an ancestral vestige, this structure may cor- 
respond to an embryonic adaptation (43). Furthermore, the subplate is important in many aspects of the development of the cortical plate (44). Comparative studies $(45,46)$ of the cerebral cortex of reptiles and of mammals have concluded that cells in the inferior layers of the isocortex (output layers $\mathrm{V}$ and VI) resemble most the cells of the reptilian cortex. However, in the reptile there are no cell types strictly comparable to those in the granular layer (IV, thalamo-recipient) and supragranular layers (II and III, involved in local and cortico-cortical connections) of the isocortex. Thus, granular and supragranular layers may be an evolutionary acquisition of the mammalian brain. Since these layers are also the latest produced cells of the isocortex, this may imply an additional developmental step in the development of the mammalian cortex. Related evidence is the fact that deep cortical layers mostly derive from the ventricular zone (the deepest, proliferative region of the hemisphere), and they are characterized by the presence of the marker Otx 1 . Cells belonging to supragranular layers derive from the subventricular zone (which is above the ventricular zone), and are positive to the Svet 1 gene. In the cortex of the small eye mouse ( $\operatorname{Pax} 6$ mutant), only deep layers are formed and the Svet 1-positive superficial layers do not develop (47). Apparently, there is an inability of the precursors of the superficial layers to differentiate and migrate into the subventricular zone. This evidence suggests that the superficial and the deep layers of the isocortex may be specified by different genetic mechanisms.

\section{Mutants of cortical development}

Several genes have been identified which participate in the regulation of cortical development. Below we will discuss two distinct signaling pathways that participate in the regulation of cell migration, which can relate to the evolutionary origin of the isocortex. These are the reelin/mDab pathway, and the Cdk5/p35 pathway (see Figure 5).

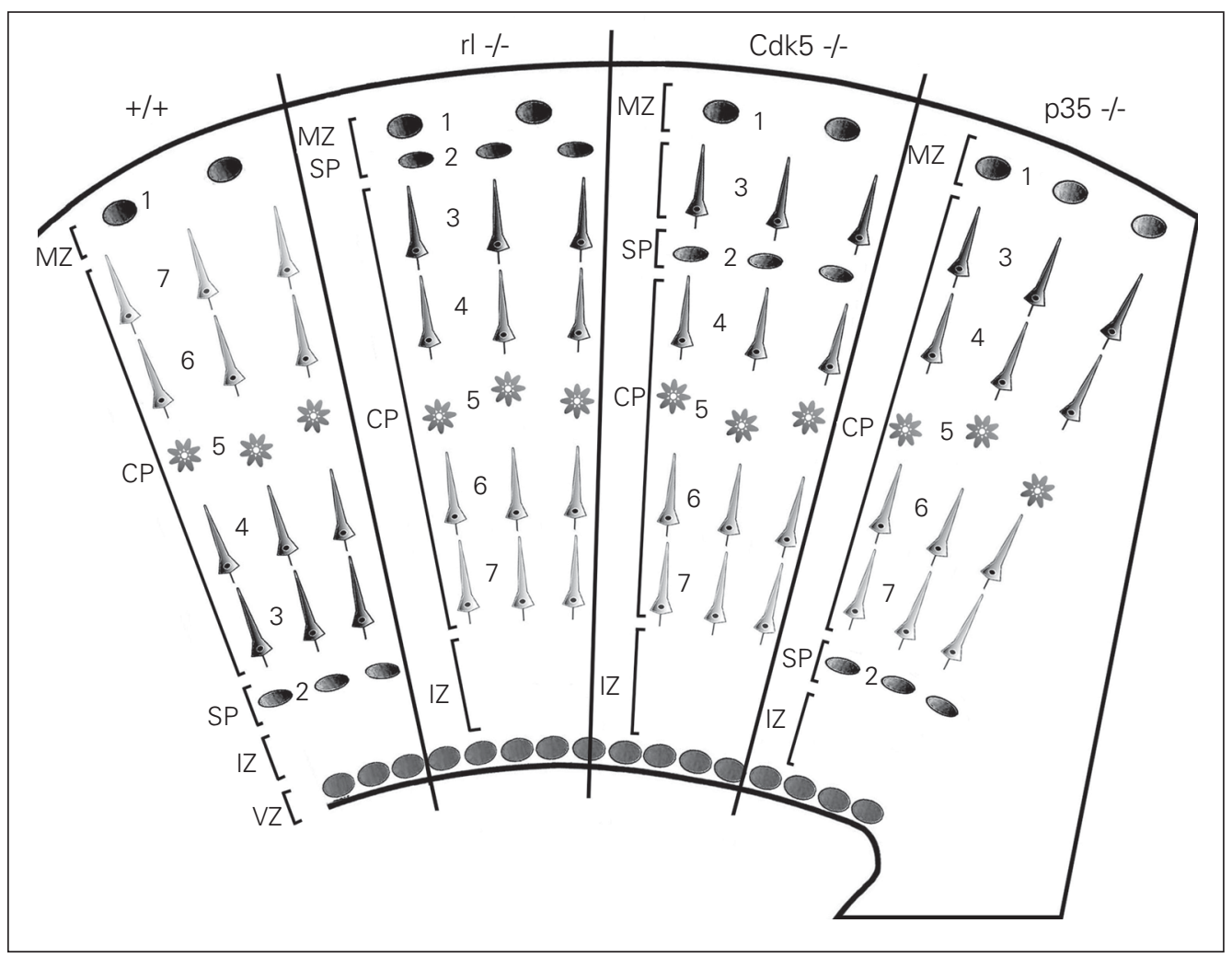

Figure 5. Cortical organization in some mutants of cortical cell migration. +/+, normal mouse; $\mathrm{rl}-/$-, reeler mouse; Cdk5 - - , cyclin-dependent kinase 5; p35 -/, p35 mutant. CP, cortical plate; IZ, intermediate zone; $M Z$, marginal zone; SP, subplate; VZ, ventricular zone. Numbers indicate order of arrival of cells to the cortex. 
The reeler mutant is characterized by an inverse layering of the cortex, in which cells normally belonging to deep layers are located superficially, and cells normally destined to superficial layers are located deeply. An important characteristic of the isocortex of the reeler mouse which is not always emphasized enough is that in the adult, cortical plate cells are located immediately below the subpial surface, there being no cellpoor layer I in this mutant. This does not happen in other regions of this mutant like the dentate gyrus and the hippocampus, in which a superficial marginal zone can be observed. The reeler phenotype is caused by mutations in a gene coding for an extracellular glycoprotein called reelin, which is secreted by the Cajal-Retzius cells of layer I (48). Reelin binds to several receptors in the migrating cells (including $\mathrm{N}$-cadherin receptors, VLDL receptors and integrin receptors), and triggers an intracellular cascade mediated by the protein mDab. Reelin is a serine protease (49), and it has been postulated that this proteolytic activity is central to its neuronal function. One hypothesis (5052 ) is that normally, by binding to these receptors, reelin detaches the neuron from the radial glia that serves as a substrate for migration, consequently arresting migration of the cell. The separation of the cell from the radial glia permits younger cells to migrate past the older neurons, using the same radial glia as a substrate, until they contact reelin molecules as they get to the border of layer I. In this way, younger cells are able to migrate past older ones, generating the inside-out neurogenetic gradient. If reelin is inactive as in the reeler mouse, neurons do not detach from the radial glia, and keep migrating until they accumulate in the subpial layer. Presumably, the inverted outsidein gradient of the reeler is produced because younger cells are not able to migrate past the older neurons that remain attached to the radial glia. In the reeler mutant, neurons are unable to migrate through the subplate and accumulate below it, possibly because the subplate cells remain attached to the radial glia. As mentioned, and perhaps due to the death of the preplate cells, in the adult, reeler cells of the cortical plate are seen reaching the subpial layer, that is, they penetrate into the marginal zone, possibly by compressing cells of the preplate or by migrating into it once the preplate cells die. It was recently observed that some of the wild characters were rescued by artificially expressing ectopic reelin in the ventricular zone of reeler mice (53). One of the most notable rescued characters was the ability of cortical plate cells to split the preplate; that is, they are able to migrate through the subplate and locate above it. However, in the cortical plate, most cells still show an inverted lamination and they migrate into the marginal zone as in the standard reeler mutant. Interestingly, ectopically expressed reelin binds to migrating cells and triggers the $\mathrm{mDab}$ intracellular cascade. In general, these results are rather hard to interpret clearly. It could be that reelin receptors are not critical for cell movement in early stages, which would explain why early-migrating cells do not arrest migration once in contact with ectopic reelin. Another possibility is that the levels of ectopically expressed reelin are too low to block migration. In addition, ectopic reelin might somehow help detaching the subplate neurons from the radial glia, allowing cortical plate cells to move through it. It could also be that ectopic reelin interacts with some factors that prevent cellular migration across the subplate. However, this might be insufficient to permit cells to migrate through previously formed layers within the cortical plate, since the inside-out neurogenetic gradient is not restored in these mice. In any case, these results indicate that the role of reelin may be more complex than has been previously thought. In this context, another recent finding indicates that reelin signaling is necessary for the migration of neural stem cells away from the ventricular zone 
(54), which opens the possibility that this molecule may have some role in early migrational stages.

The next set of mutations relate to the cyclin-dependent kinase Cdk5 and its activator p35. Mutants for Cdk5 have a phenotype somewhat similar to the reeler one in that cells arrange in an outside-in pattern and most neurons arrange below the subplate, forming an underplate (actually, many cells can be observed in the intermediate zone, forming clusters that apparently displace very slowly along the radial glia; 55). However, there are two important differences with the reeler phenotype: first, layer VI cells (the earliest produced) are able to cross the subplate, correctly splitting the preplate, and second, in the adult there is a normal layer I, indicating that cells do not penetrate into the marginal zone and thus may respond normally to reelin. The p 35 mutant has a milder phenotype, as in this animal cortical plate cells migrate better and all are able to move past the subplate, splitting the preplate. However, within the cortical plate, cells arrange in an inverted outside-in pattern. As in the Cdk5 mutant, in the adult p35 mutant there is a normal layer I. Summarizing, early-produced layer VI cells do not need either Cdk5 or p35 to reach their proper position and cross the subplate. Late-produced cells from layers II-V need Cdk5 but not p35 to cross the subplate, and need both $\mathrm{Cdk} 5$ and p35 to cross through previous cortical layers within the cortical plate. There is another Cdk5 activator, p39, whose mutation produces a very mild phenotype. The p35/p39 double mutant has been claimed to totally reproduce the phenotype of the Cdk5 mutant (56). This suggests that Cdk5, activated by p39, helps cells from layers II-V to migrate through the intermediate zone and to cross the subplate. In this context, it has been observed that the POU domain transcription factors Brn 1 and $B r n 2$ critically control the initiation of radial migration by regulating the Cdk5-regulatory elements p35 and p39 (57).
Recently, two modes of cell migration have been observed in the developing cerebral cortex (58): one is called translocation, and is observed in early-produced cells of the cerebral cortex, including those of layer VI, and also in the latest stages of migration of late-produced cells within the cortical plate. In this modality, the apical process of the cell contacts the subpial layer, and the cell body is dragged toward the surface while the apical process shortens. The other mode, locomotion, consists of the continuous lengthening and shortening of the apical process as the cell migrates along the radial glia. It is highly interesting that in the Cdk5 and the p35 mutants, only cells that move by locomotion are affected; cells in layer VI and other early-produced cells that migrate mainly by translocation are unaffected (51, 59). This suggests that Cdk5 and its activators p35 and p39 participate in helping migration by locomotion. It is then possible that $\mathrm{Cdk} 5$, activated by $\mathrm{p} 35$, allows migrating neurons to move by locomotion within the cortical plate, thus allowing the generation of the inside-out gradient. It has also been found that Cdk5 and p35 inhibit Ncadherin-dependent cell aggregation in migrating cortical neurons, which may also be part of a mechanism that permits migrating neurons to move past other cells within the cortical plate (50-52).

\section{Evolutionary origin of the inside-out neurogenetic gradient}

Considering this evidence, we postulated (50-52) that there was a hypothetical mammal-like reptile with an outside-in pattern of cell migration like that of reptiles. Perhaps in this animal many cells migrated by using the translocation mode of cell migration, and cells also arranged in an outside-in manner. Associated with the early expansion and increase in cell number of the evolving brain (origin of more superficial layers), the Cdk5 pathway may have been a key factor that 
permitted long-distance migration by locomotion of the new cell types. Furthermore, p35 was probably fundamental to permit cells to migrate past older cells in the cortical plate, generating the outside-in gradient. However, these cells activated by Cdk5 may have needed to be prevented from migrating into the marginal zone. The role of reelin may have precisely been to stop these cells before they penetrate into layer I. Although recent findings by Magdaleno et al. (53) and Kim et al. (54) indicate that the developmental role of reelin may be more complex than a simple stop signal, the evidence points to a critical role of reelin in the final stages of neuronal migration, perhaps specifically in the generation of a cell-free marginal zone. It is interesting that reelin is quite poorly expressed in the developing cortex of reptiles (60), and that in the reeler mutant there is a normal layer I in the hippocampus and dentate gyrus, suggesting that in all these cases there is some other factor beside reelin that prevents cells from entering the marginal zone.

Yet there are some open questions. One is that, like other cortical plate cells, the cells produced early from layer VI (which are Cdk5-independent) seem to depend on reelin to avoid penetrating into the marginal zone. If these cells migrate according to a mechanism different from the younger cells of the cortical plate, they might not depend on reelin as a stop signal. Rather, they might be prevented from migrating into the marginal zone by some mechanism similar to that found in reptiles (and perhaps also in the hippocampus and dentate gyrus). This might be because the ancestral stop signal was totally replaced by reelin, and is no longer present in the isocortex. Another point is that layer $\mathrm{V}$ cells may actually represent an intermediate stage between the new phenotypes of the granular and supragranular layers and those of the earliest-produced cells of layer VI. Layer V cells are produced relatively early in development, derive from the ven- tricular zone, and have phenotypes similar to those of the reptilian cortex. However, they do depend on $\mathrm{Cdk} 5 / \mathrm{p} 35$ to migrate and reach their proper position.

\section{Some why questions}

Why did all these changes in isocortical organization come about? Why was there an inverted layering in the isocortex, and why do axons have a radial entrance to it? Up to this point, we have discussed the developmental mechanisms involved in isocortical origins, but to make sense of the evolutionary transition, it is important to discuss the adaptive or functional context in which this process occurred. Below, we will outline some hypotheses attempting to shed some light on this issue.

As discussed before, the dorsal cortex of reptiles has most afferents located in the marginal zone, and the apical dendrites of the pyramidal cells make synapses with these afferents. In most mammals, afferents enter the cortex radially from the underlying white matter. However, in some so-called primitive mammals like the hedgehog, afferents enter radially to the cortex, but eventually they penetrate into layer I and run tangentially for some distance (6). This "pseudotangential" organization may represent a vestigial reminder of the ancestral condition of reptiles.

Now let us consider an hypothetical ancestral situation. If afferents were located in the superficial zone, and the cerebral cortex was expanding in thickness, an outside-in gradient like that of the reptilian cortex would have left the new cells, involved in intracortical processing, too far from the cortical afferents in layer I (they would be separated from them by the early-produced, output cells). The solution to this problem was that the new cell types migrated past the older cells, and thus were able to make direct synaptic contacts with the afferents in layer I, in this manner favoring cortical processing capacity. 
In a next stage, the early mammalian cortex began to expand in the tangential direction. In such a situation, tangentially running afferents would have to run increasingly long distances from the lateral aspect of the cortex (where they enter into the marginal zone) to the site in which they establish their sites of termination. In addition, the marginal zone might have been soon crowded with thalamocortical axons. Perhaps the simplest solution, which possibly occurred very early in cortical evolution, was to run axons into the subcortical white matter and let them enter the cortex radially. In this process, the origin or differentiation of the subplate may have been a pivotal element in attracting axons to the subcortical matter. During development, subplate cells send pioneer axons which establish contact with thalamic axons, guiding them into the subplate and allowing them to establish transient synaptic contacts with subplate cells before the cortical plate is ready to receive these axons. Later, thalamic axons are able to penetrate radially into the isocortex, possibly aided by reciprocal connections between the subplate and cortical layer IV (44). According to these hypotheses, the participation of the subplate in axonal guidance and its elaboration into a complex cell layer may have occurred in evolution after the origin of the superficial layers of the isocortex. Nevertheless, during development the subplate appears earlier than the cortical plate. The lack of concordance between the proposed phylogenetic sequence and the ontogenetic processes may be explained by the fact that the subplate is an embryonic adaptation, rather than an adult adaptation like the superficial isocortical layers.

In this way, the inside-out neurogenetic gradient may have been associated with the radial expansion of the isocortex (origin of the superficial layers), while the radial organization of thalamic inputs was possibly more related to the tangential expansion of the early isocortex. In this latter process, the subplate may have been a fundamental element in attracting axons through a shortcut via the developing intermediate zone.

\section{Conclusions}

This paper has attempted to provide a coherent scenario for the early evolution of the isocortex. The first main point expressed here is that the isocortex possibly arose from expansion of the ancestral dorsal cortex, and may have not arisen from the DVR. The possibility of the ventral pallium (DVR) contributing to the isocortex depends on demonstrating a sufficiently massive migratory process from the former to the latter, something that has not been observed. Perhaps one possibility to reconcile the two hypotheses would be that, although the ventrolateral isocortex may derive mostly from the dorsal pallium, a population of thalamo-receptive cells has migrated dorsally from the ventral pallium, dragging with them the collothalamic afferents. In any case, this alternative also relies on the demonstration of a tangential migratory pathway from the ventral pallium to the dorsal pallium.

Regardless of this controversy, the brains of mammals are differently organized than those of reptiles, in that all sensory pathways converge in one structure, the isocortex, and then are all projected into both the hippocampus and the amygdala. This differs from the organization of the reptilian brain, in which there are two types of sensory pathways that remain largely separate. It is possible that the development of olfaction in early mammals placed emphasis on hippocampal-olfactory cortex circuits for processing spatial information, which promoted the development of the lemnothalamic systems and the confluence of the collothalamic pathways into the hippocampus. Although this latter concept is compatible with both the recapitulation and the outgroup hypotheses, it is more parsimoniously coherent with the outgroup hypothesis in that it requires less 
dramatic developmental transformations.

The second main point of this paper is that the superficial isocortical laminae are evolutionarily new components of the isocortex, and their origin, together with the tangential expansion of the early isocortex, triggered the development of the inverted neurogenetic gradient of mammals and the radial entrance of thalamocortical axons, respectively. At least two signaling pathways, one mediated by reelin and the other by Cdk5/p35, may have been fundamental in the origin of the inside-out gradient. Particularly, the activator p35 was fundamental in allowing migrating cells to locomote within the cortical plate, thus producing the characteristic inside-out gradient of the isocortex, while reelin was recruited to prevent cells from migrating into the marginal zone.

These considerations also raise several intriguing questions. For example, a lot more research is needed into the ventral pallium and its developmental fate. It will also be interesting to study the possibility of dorsalizing, or ventralizing factors, in telencephalic development, like BMP 4 and Sonic hedgehog, which are respectively known as dorsalizing and ventralizing factors in the neural tube and in the telencephalon. Furthermore, the point that lemnothalamic and collothalamic pathways are probably largely separated in reptiles may imply that these animals process information somewhat differently than mammals do.

We believe that embryonic processes may give important insights into the evolutionary mechanisms involved in the origin of the mammalian brain. However, these need to be complemented with functional considerations providing integrative scenarios which attempt to reconstruct the historical processes as accurately as possible. Without question, this is a risky endeavor, but on the other hand provides new questions that may direct future comparative research.

\section{References}

1. Carroll RL (1988). Vertebrate Paleontology and Evolution. Freeman Press, New York, NY, USA.

2. Hopson JA (1979). Paleoneurology. In: Gans CC, Northcutt RG \& Ulinski PS (Editors), Biology of the Reptilia. Vol. 4. Academic Press, New York, NY, USA.

3. Rowe T (1996). Coevolution of the mammalian middle ear and neocortex. Science, 273: 651-654.

4. Wang $Y, H u ~ Y$, Meng $J$ \& Chuankui $L$ (2001). An ossified Meckel's cartilage in two cretaceous mammals and origin of the mammalian middle ear. Science, 294: 357-361.

5. Neary TJ (1990). The pallium of anuran amphibians. In: Jones EG \& Peters A (Editors), Cerebral Cortex. Vol. 8B. Plenum Press, New York, NY, USA.

6. Ten Donkelaar HJ (1998). Reptiles. In: Nieuwenhuys R, Ten Donkelaar HJ \& Nicholson C (Editors), The Central Nervous System of Vertebrates. SpringerVerlag, Berlin, Germany.

7. Northcutt RG \& Kaas JH (1995). The emergence and evolution of mammalian neo- cortex. Trends in Neurosciences, 18: 373379.

8. Karten HJ (1969). The organization of the avian telencephalon and some speculations on the phylogeny of the amniote telencephalon. Annals of the New York Academy of Sciences, 167: 164-179.

9. Karten HJ (1997). Evolutionary developmental biology meets the brain: the origins of mammalian neocortex. Proceedings of the National Academy of Sciences, USA, 94: 2800-2804.

10. Butler $A B$ (1994). The evolution of the dorsal pallium in the telencephalon of amniotes: cladistic analysis and a new hypothesis. Brain Research. Brain Research Reviews, 19: 66-101.

11. Medina L \& Reiner A (2000). Do birds possess homologues of mammalian primary visual, somatosensory and motor cortices? Trends in Neurosciences, 23: 1-12.

12. Aboitiz $F$ (1992). The evolutionary origin of the mammalian cerebral cortex. Biological Research, 25: 41-49.

13. Aboitiz F (1995). Homology in the evolution of the cerebral hemispheres: the case of reptilian dorsal ventricular ridge and its possible correspondence with mammalian neocortex. Journal of Brain Research, 4: 461-472.

14. Wilczynski W \& Northcutt RG (1983). Connections of the bullfrog striatum: afferent organization. Journal of Comparative Neurology, 214: 321-332.

15. Bruce LL \& Neary TJ (1995). The limbic system of tetrapods: a comparative analysis of cortical and amygdalar populations. Brain, Behavior and Evolution, 46: 224234.

16. Guirado S, Dávila JC, Real MA \& Medina L (2000). Light and electron microscopic evidence for projections from the thalamic nucleus rotundus to targets in the basal ganglia, the dorsal ventricular ridge, and the amygdaloid complex in a lizard. Journal of Comparative Neurology, 424: 216232.

17. Yoon MS, Puelles L \& Redies C (2000). Formation of cadherin-expressing brain nuclei in diencephalic and alar plate divisions. Journal of Comparative Neurology, 427: $461-480$ 
18. Montero V (1993). Retinotopy of cortical connections between the striate cortex and extrastriate visual areas in the rat. Experimental Brain Research, 94: 1-15.

19. Rosa MGP \& Krubitzer LA (1999). The evolution of visual cortex: where is V2? Trends in Neurosciences, 22: 242-248.

20. Aboitiz $F$ (1999). Evolution of isocortical organization. A tentative scenario including roles of reelin, p35/cdk5 and the subplate zone. Cerebral Cortex, 9: 655661.

21. Smith-Fernández A, Pieau $C$, Repérant J, Boncinelli E \& Wassef M (1998). Expression of the Emx-1 and Dlx-1 homeobox genes define three molecularly distinct domains in the telencephalon of mouse, chick, turtle and frog embryos: implications for the evolution of telencephalic subdivisions in amniotes. Development, 125: 2099-2111.

22. Puelles L, Kuwana E, Puelles E \& Rubenstein JLR (1999). Comparison of the mammalian and avian telencephalon from the perspective of gene expression data. European Journal of Morphology, 37: 139150.

23. Puelles L, Kuwana E, Puelles E, Bulfone A, Shimamura K, Keleher J, Smiga S \& Rubenstein JLR (2000). Pallial and subpallial derivatives in the embryonic chick and mouse telencephalon, traced by the expression of the genes Dlx-2, Emx-1, Nkx2.1, Pax-6 and Tbr-1. Journal of Comparative Neurology, 424: 409-438.

24. Holmgren N (1925). Points of view concerning forebrain morphology in higher vertebrates. Acta Zoologica, 6: 413-477.

25. Parnavelas JG (2000). The origin and migration of cortical neurones: new vistas. Trends in Neurosciences, 23: 126-131.

26. Marin O \& Rubenstein JL (2001). A long, remarkable journey: tangential migration in the telencephalon. Nature Reviews. Neuroscience, 2: 780-790.

27. Deng J \& Elberger AJ (2001) The role of pioneer neurons in the development of mouse visual cortex and corpus callosum. Anatomy and Embryology, 204: 437-453.

28. Chan CH, Godinho LN, Thomaidou D, Tan SS, Gulisano M \& Parnavelas JG (2001). Emx1 is a marker for pyramidal neurons of the cerebral cortex. Cerebral Cortex, 11: 1191-1198.

29. Cobos I, Puelles L \& Martinez S (2001). The avian telencephalic subpallium originates inhibitory neurons that invade tangentially the pallium (dorsal ventricular ridge and cortical areas). Developmental Biology, 239: 30-45.

30. Chapouton P, Gärtner A \& Götz M (1999).
The role of Pax6 in restricting cell migration between developing cortex and basal ganglia. Development, 126: 5569-5579.

31. Stoykova A, Treichel D, Hallonet M \& Gruss $P$ (2000). Pax 6 modulates the dorsoventral patterning of the mammalian telencephalon. Journal of Neuroscience, 20: 8042-8050

32. Britto J, Tannahill D \& Keynes R (2002). A critical role for sonic hedgehog signaling in the early expansion of the developing brain. Nature Neuroscience, 5: 103-110.

33. Day LB, Crews D \& Wilczynski W (2001). Effects of medial and dorsal cortex lesions on spatial memory in lizards. Behavioural Brain Research, 118: 27-42.

34. Eichenbaum $\mathrm{H}$ (1998). Using olfaction to study memory. Annals of the New York Academy of Sciences, 855: 657-669.

35. Ulinski PS (1990). The cerebral cortex of reptiles. In: Jones EG \& Peters A (Editors), Cerebral Cortex. Vol. 8B. Plenum Press, New York, NY, USA.

36. Ulinski PS (1983). Dorsal Ventricular Ridge: A Treatise on Brain Organization in Reptiles and Birds. John Wiley \& Sons, New York, NY, USA.

37. Goffinet AM, Daumerie C, Langerwerf B \& Pieau C (1986). Neurogenesis in reptilian cortical structures: ${ }^{3} \mathrm{H}$-thymidine autoradiographic analysis. Journal of Comparative Neurology, 243: 106-116.

38. Rakic P (1974). Neurons in rhesus monkey: systematic relation between time of origin and eventual disposition. Science, 183: 425-427.

39. Rakic P (1995). A small step for the cell, a giant leap for mankind: a hypothesis of neocortical expansion during evolution. Trends in Neurosciences, 18: 383-388.

40. Nacher J, Ramírez C, Molowny A \& LópezGarcía C (1996). Ontogeny of somatostatin immunoreactive neurons in the medial cerebral cortex and other cortical areas of the lizard Podarcis hispanica. Journal of Comparative Neurology, 374: 118-135.

41. Cordery P \& Molnár Z (1999). Embryonic development of connections in turtle pallium. Journal of Comparative Neurology, 413: 26-54.

42. Marín-Padilla M (1978). Dual origin of the mammalian neocortex and evolution of the cortical plate. Anatomy and Embryology, 152: 109-126.

43. Kostovic I \& Rakic P (1990). Developmental history of the transitional subplate zone in visual and somatosensory cortex of macaque monkey and human brain. Journal of Comparative Neurology, 297: 441470.

44. Allendoerfer KL \& Shatz CJ (1994). The subplate, a transient neocortical structure: its role in the development of connections between thalamus and cortex. Annual Review of Neuroscience, 17: 185218.

45. Ebner FF (1969). A comparison of primitive forebrain organization in metatherian and eutherian mammals. Annals of the New York Academy of Sciences, 167: 241-257.

46. Reiner A (1991). A comparison of neurotransmitter-specific and neuropeptidespecific neuronal cell types present in the dorsal cortex of reptiles with those present in the isocortex of mammals. Brain, Behavior and Evolution, 38: 53-91.

47. Tarabykin V, Stoykova A, Usman N \& Gruss P (2001). Cortical upper layer neurons derive from the subventricular zone as indicated by Svet1 gene expression. Development, 128: 1983-1993.

48. Curran T \& D'Arcangelo G (1998). Role of reelin in the control of brain development. Brain Research Reviews, 26: 285-294.

49. Quattrocchi CC, Wannenes F, Persico AM, Ciafré SA, D'Arcangelo G, Farace MG \& Keller $F$ (2002). Reelin is a serine protease of the extracellular matrix. Journal of Biological Chemistry, 277: 303-309.

50. Aboitiz F (2001). The evolution of cortical development. Trends in Neurosciences, 24: 202-203.

51. Aboitiz F, Morales D \& Montiel J (2001). The inverted neurogenetic gradient of the mammalian isocortex: development and evolution. Brain Research Reviews, 38: 129-139.

52. Aboitiz F, Montiel J \& López J (2002). A hypothesis on the early evolution of the develoment of the isocortex. Brain Research Bulletin, 57: 481-483.

53. Magdaleno $S$, Keshvara L \& Curran $T$ (2002). Rescue of ataxia and preplate splitting by ectopic expression of Reelin in reeler mice. Neuron, 33: 573-586.

54. Kim HM, Qu T, Kriho V, Lacor P, Smalheiser N, Pappas GD, Guidotti A, Costa E \& Sugaya K (2002). Reelin function in neural stem cell biology. Proceedings of the National Academy of Sciences, USA, 99: 4020-4025.

55. Pearlman AL, Faust PL, Hatten ME \& Brunstrom JE (1998). New directions for neuronal migration. Current Opinion in Neurobiology, 8: 45-54.

56. Ko J, Humbert S, Bronson RT, Takahashi S, Kulkarni AB, Li E \& Tsai LH (2001). p35 and p39 are essential for cyclin-dependent kinase 5 function during neurodevelopment. Journal of Neuroscience, 21: 6758-6771. 
57. McEvilly RJ, de Diaz MO, Schonemann MD, Hooshmand F \& Rosenfeld MG (2002). Transcriptional regulation of cortical neuron migration by POU domain factors. Science, 295: 1528-1532.

58. Nadarajah B, Brunstrom JE, Grutzendler J, Wong RO \& Pearlman AL (2001). Two modes of radial migration in early development of the cerebral cortex. Nature Neuroscience, 4: 143-150.

59. Gilmore EC \& Herrup K (2001). Neocortical cell migration: GABAergic neurons and cells in layers I and VI move in a cyclindependent kinase 5-independent manner.
Journal of Neuroscience, 21: 9690-9700.

60. Bar I, Lambert de Rouvroit C \& Goffinet A (2000). Reelin mRNA expression during embryonic brain development in the turtle Emys orbicularis. Journal of Comparative Neurology, 413: 463-479. 